\title{
Fractal Square Zone Plates
}

\author{
A. Calatayud ${ }^{1}$, V. Ferrando ${ }^{1,2}$, F. Giménez ${ }^{3}$, \\ W.D. Furlan ${ }^{2}$, G. Saavedra ${ }^{2}$, and J.A. Monsoriu ${ }^{1 *}$ \\ ${ }^{1}$ Centro de Tecnologías Físicas, Universitat Politècnica de València, E-46022 Valencia, \\ Spain \\ ${ }^{2}$ Departamento de Óptica, Universitat de València, E-46100 Burjasstot (Valencia), \\ Spain \\ ${ }^{3}$ I.U. Matemática Pura y Aplicada, Universitat Politècnica de València, E-46022 \\ Valencia, Spain
}

\begin{abstract}
In this paper we present a novel family of zone plates with a fractal distribution of square zones. The focusing properties of these fractal diffractive lenses coined fractal square zone plates are analytically studied and the influence of the fractality is investigated. It is shown that under monochromatic illumination a fractal square zone plate gives rise a focal volume containing a delimited sequence of two-arms-cross pattern that are axially distributed according to the self-similarity of the lens.
\end{abstract}

Keywords: Diffraction, diffractive lens, zone plates, fractals

"E-mail address: jmonsori@fis.upv.es 


\section{Introduction}

Diffractive optics has found a great number of new applications in the last few years, satisfying the increasing demand of more compact, light-weight, and costeffective optical systems and components. With the rapid development of photonic technology, a wide range of applications arises also in areas where conventional refractive optics do not provide good solutions or where it is even impossible to use it, as for example, in some branches of Ophthalmology [1], in THz Imaging [2], and in Xray Microscopy [3]. Furthermore, with the availability of spatial light modulators technology, variable geometry of generated wavefronts becomes possible and DOEs can be recorded in real-time in recyclable recording media. This speeds up the development of new ideas in science and technology as cost-efficient rapid prototyping of DOEs and also in real time applications. Following this trend, a few years ago, we presented fractal zone plates (FZPs) [4] as new promising diffractive lenses with interesting focusing [5] and imaging properties [6]. A FZP is characterized by its fractal profile along the square of the radial coordinate. The main feature of a FZP arises from its ability to produce multiple foci distributed in a fractal way along the optical axis reproducing the selfsimilarity of the FZP itself.

Square zone plates (SZPs) [7, 8] are another kind of promising diffractive optical elements which are the result of the combination of two linear Fresnel zone plates [9]. Thus, its resulting structure presents alternate transparent and opaque square zones with side length $\sqrt{j} b$, where $b$ is the side length of the first zone and $j$ is the number of the zone. Under a monochromatic plane wave illumination this configuration produces a focalization pattern with a cross-like irradiance distribution. SZPs have exhibited applications in precision alignment systems [10], in infrared antennas [11] and more recently have been combined with spiral phase masks for generating hollow beams [12]. 
In this work we introduce the concept of fractal square zone plates (FSZP), i.e. zone plates with a fractal distribution of square zones. Some practical considerations about the design of this type of SZP are investigated, taking into account the physical limits imposed by the different construction parameters. Finally, the influence on the axial and transverse irradiance produced by the fractality of these lenses is numerically evaluated and compared with the response of conventional SZPs.

\section{FSZPs design.}

Our proposal for a FSZP is based on the triadic Cantor set shown in Fig. 1(a). The first step in the construction procedure consists in defining a straight-line segment of unit length called initiator (stage $S=0$ ). Next, at stage $S=1$, the generator of the set is constructed by dividing the segment in three equal parts of length $1 / 3$ and removing the central one. Following this procedure in subsequent stages $S=2,3, \ldots$ is easy to see that, in general, at stage $S$ there are $2^{S}$ segments (each one of length $3^{-S}$ ) separated by $2^{S}-1$ gaps. In Fig. 1(a), only the four first stages are shown for clarity. Note that the $S$-th stage of the Cantor set can be interpreted as a quasiperiodic distribution of segments which can be obtained by removing some segments in the finite periodic distribution as shown in Fig. 1(b). This periodic distribution at stage $S$ has $\left(3^{S}+1\right) / 2$ segments with $\left(3^{S}\right.$ 1)/2 gaps being the total number of elements (segments+gaps) $N=3^{S}$.

(a) Fractal structure

(b) Periodic structure

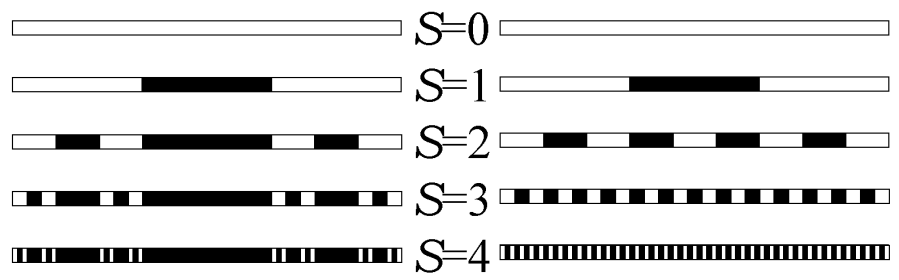

Fig. 1. Comparison between (a) the triadic Cantor set and (b) the equivalent periodic structure generated at different stages $S$. 
It is interesting, for our purposes, to note that triadic Cantor set in Fig. 1(a) can also codified using an array of binary elements [13]. The generating array for $S=1$ is $\{101\}$, where " 1 " represent a white segment and "0" a black segment in Fig. 1. The Cantor array is generated recursively by replacing $\{1\}$ by $\{101\}$ and $\{0\}$ by $\{000\}$ at each stage of the construction of the fractal structure. Then, the corresponding array at $S=2$ would be $\{101000101\}$ and the equivalent periodic array $\{101010101\}$.

A FSZP can be constructed in a similar way to a SZP; i.e., as a sequence of square zones with side length $\sqrt{j} b$ but defining the transmittance $t_{j}$ of the $j$-th zone as the $j$-th element of the fractal binary array where $t_{j}=1$ for transparent zones and $t_{j}=0$ for opaque zones. The resulting structure for $S=3$ is shown in Fig. 2(a) and Fig. 2(b) shows the equivalent SZP with the same focal length. Note that a FSZP can be considered as a SZP but with some missing clear square zones.

In mathematical terms, the transmittance function for both kinds of lenses can be written with the same mathematical expression,

$p(x, y)=\sum_{j=1}^{N} t_{j}\left[\operatorname{rect}\left(\frac{x}{a \sqrt{j / N}}\right) \operatorname{rect}\left(\frac{y}{a \sqrt{j / N}}\right)-\operatorname{rect}\left(\frac{x}{a \sqrt{(j-1) / N}}\right) \operatorname{rect}\left(\frac{y}{a \sqrt{(j-1) / N}}\right)\right]$,

where $N$ is the total number of zones and the length of the outer zone is $2 a$.
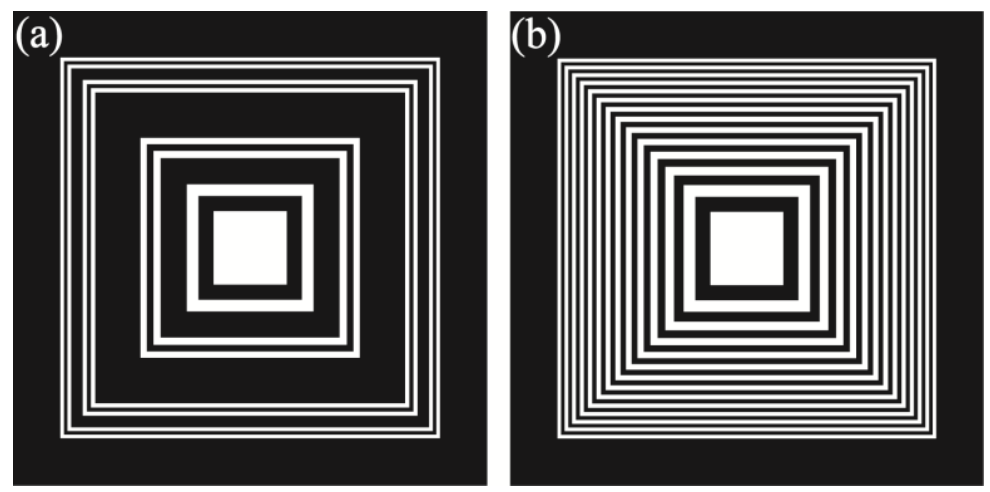

Fig. 2. Comparison between (a) a FSZP and (b) the equivalent SZP ( $S=3)$. 


\section{Focusing properties of FSZPs.}

Let us consider the irradiance provided by a square pupil of length $2 a$, and amplitude transmittance $p(x, y)$, when it is illuminated by a monochromatic plane wave of wavelength $\lambda$. Within the Fresnel approximation the diffracted field at a given point $(x, y, z)$, where $z$ is the axial distance from the pupil plane, can be expressed by

$I(x, y, z)=\frac{1}{\lambda^{2} z^{2}}\left|\iint_{-\infty}^{\infty} p\left(x_{o}, y_{o}\right) e^{\frac{-i \pi}{\lambda z}\left(x_{o}^{2}+y_{o}^{2}\right)} e^{\frac{i 2 \pi}{\lambda z}\left(x x_{o}+y y_{o}\right)} d x_{o} d y_{o}\right|^{2}$

If the transmittance of the pupil is defined in terms of the normalized variables, $\left\{\bar{x}_{o}, \bar{y}_{o}\right\}=\left\{x_{o} / a, y_{o} / a\right\}$, then the irradiance can be re-written as,

$I(\bar{x}, \bar{y}, u)=4 u^{2}\left|\iint_{-1}^{1} q\left(\bar{x}_{o}, \bar{y}_{o}\right) e^{-i 2 \pi u\left(\bar{x}_{o}^{2}+\bar{y}_{o}^{2}\right)} e^{i 2 \pi u\left(2 \bar{x} \bar{x}_{o}+2 \bar{y} \bar{y}_{o}\right)} d \bar{x}_{o} d \bar{y}_{o}\right|^{2}$.

where $q\left(\bar{x}_{o}, \bar{y}_{o}\right)=p\left(a \bar{x}_{o}, a \bar{y}_{o}\right), u=a^{2} / 2 \lambda z$ is the reduced axial coordinate, and $\{\bar{x}, \bar{y}\}=\{x / a, y / a\}$ are the normalized transverse coordinates.

If we consider the diffracting square aperture of transmittance (1) in the above equation we obtain the analytical expression of the irradiance :

$$
\begin{aligned}
& I(\bar{x}, \bar{y}, u)=\frac{1}{16} \mid \sum_{j=1}^{N} t_{j}\{(\operatorname{erf}[(1+i) \sqrt{\pi u}(\sqrt{j / N}+\bar{x})]+\operatorname{erf}[(1+i) \sqrt{\pi u}(\sqrt{j / N}- \\
& \bar{x})]) \times(\operatorname{erf}[(1+i) \sqrt{\pi u}(\sqrt{j / N}+\bar{y})]+\operatorname{erf}[(1+i) \sqrt{\pi u}(\sqrt{j / N}-\bar{y})])- \\
& (\operatorname{erf}[(1+i) \sqrt{\pi u}(\sqrt{(j-1) / N}+\bar{x})]+\operatorname{erf}[(1+i) \sqrt{\pi u}(\sqrt{(j-1) / N}-\bar{x})]) \times \\
& (\operatorname{erf}[(1+i) \sqrt{\pi u}(\sqrt{(j-1) / N}+\bar{y})]+\operatorname{erf}[(1+i) \sqrt{\pi u}(\sqrt{(j-1) / N}-\bar{y})])\}\left.\right|^{2} .
\end{aligned}
$$

where $\operatorname{erf}(x)$ is the error function of the argument $x$. If we focus our attention to the values that the irradiance takes along the optical axis, Eq. (4) becomes:

$$
I(0,0, u)=\left|\sum_{j=1}^{N} t_{j}\left(\operatorname{erf}^{2}[(1+i) \sqrt{\pi u} \sqrt{j / N}]-\operatorname{erf}^{2}[(1+i) \sqrt{\pi u} \sqrt{(j-1) / N}]\right)\right|^{2}
$$

The axial irradiance of FSZPs computed for different stages of growth $S$ is shown in Fig. 3. The irradiance of the associated SZPs is shown in the same figure for comparison. Note that the scale for the axial coordinate in each step is a magnified 
version of the one in the previous step by a factor $\gamma=3$. It can be seen that the axial positions of the central lobes of the main foci coincide with those of the associated SZP. Interestingly, the irradiance produced by the FSZPshave fractal properties. In fact, the three patterns in the left part of Fig. 3 are self-similar, i.e., the axial irradiance distribution corresponding to a given FSZP of order $S=4$ is a modulated version of the irradiance distribution corresponding to the FSZP of order $S=3$. The same happens for $S=3$ and $S=2$. This means that the axial irradiance generated by a FSZP is self-similar, as it is the FSZP itself.
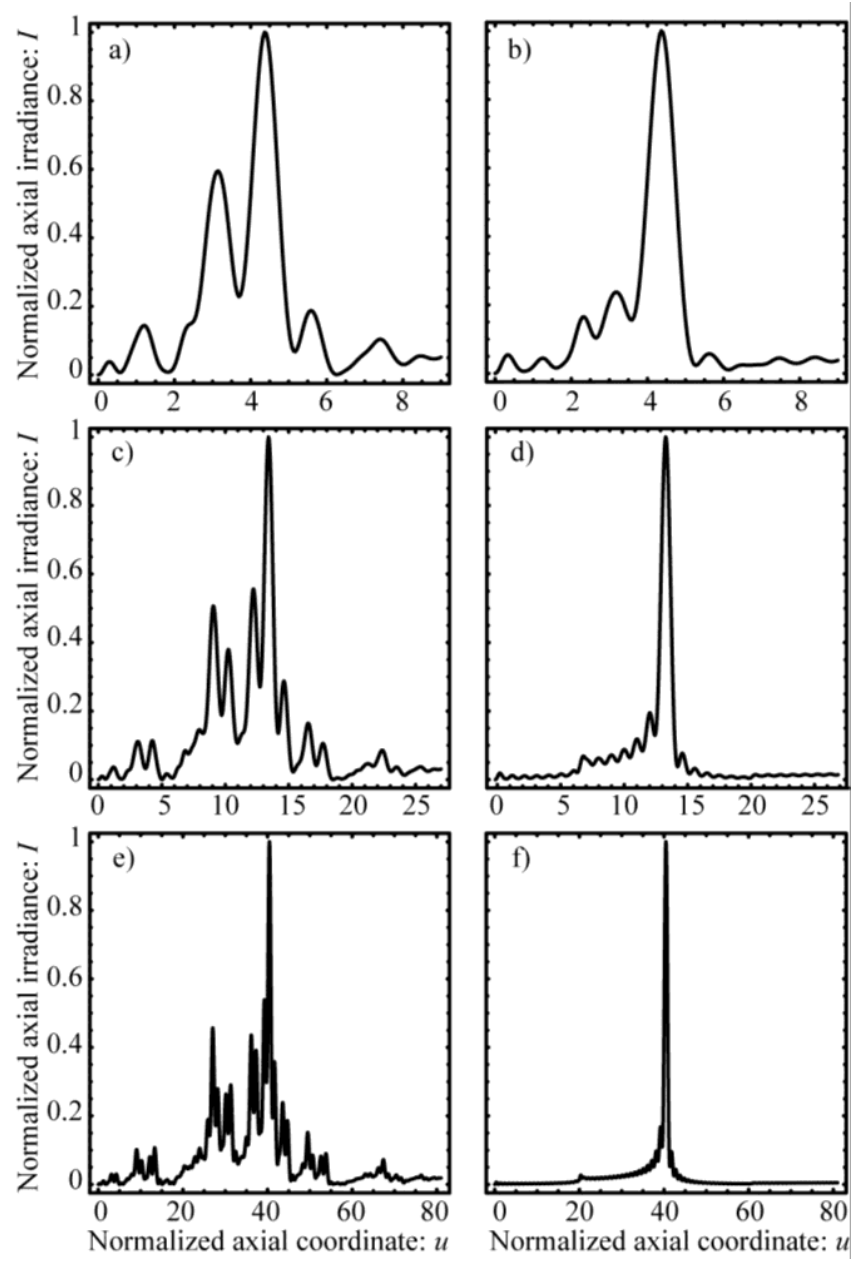

Fig.3.Normalized irradiance vs. the axial coordinate $u$ obtained for a FSZP (left part) and its associated SZP (right part) at three stages of growth, $S=2$ (upper part), $S=3$ (middle part), and $S=4$ (lower part). 
The distribution of the diffracted energy, not only in the optical axis but over the whole transverse plane is of interest for the prediction of the applications capabilities of FSZPs. Thus, a two-dimensional analysis of the diffracted intensities is required. Equation (4) has been used to calculate the evolution of the diffraction patterns for a FSZP from near field to far field. Of particular interest are the intensities at transverse planes corresponding to the different maxima of the axial irradiance. Fig. 4 shows the result obtained for $S=3$ at the main focus located at $u=13.40$ and two subsidiary foci located at $u=12.20$, and $u=9.05$. Intensities are normalized to the maximum value obtained at the main focus.

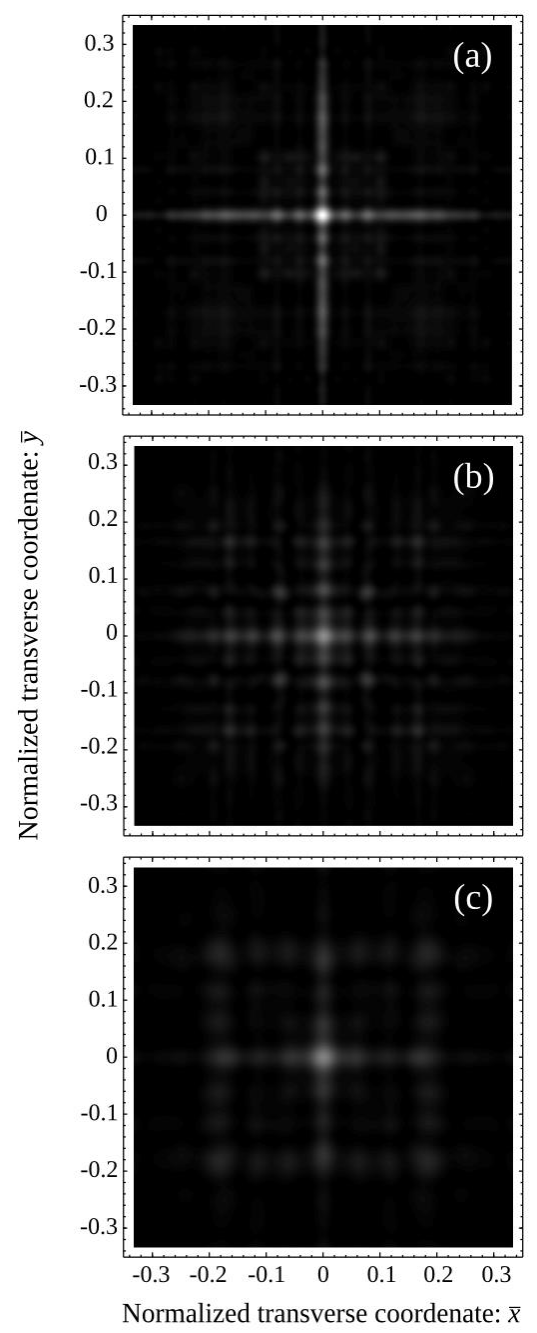

Fig. 4.Transverse diffraction patternsof a SFZP with $S=3$ at(a) the main focus located at $u=13.40$, and two subsidiary foci located at (b) $u=12.20$ and (c) $u=9.05$. 
It is shown that under monochromatic illumination a FSZP gives a main focal volume containing a two-arms-cross pattern which, at this plane, is similar to that obtained with the associated SZP (not shown). However, it can be noted that the secondary maxima at $u=12.20$, and $u=9.05$ preserves the crossed shape, and this behavior can be interpreted as an extension of depth of focus. In fact, the intensity given by the associated SZP at these planes is almost zero.

\section{Conclusions.}

A new family of diffractive lenses, coined "Fractal Square Zone Plates", has been introduced. It is shown that the distribution of square zones of theses fractal lenses is obtained through the triadic Cantor set. The focusing properties of FSZPs have been analyzed and compared with those corresponding to a conventional SZP. Under monochromatic illumination FSZPs produces a focal volume containing a delimited sequence of two-arms-cross pattern that are axially distributed according to the selfsimilarity of the lens. One potential application of these new designs of square lenses with increased depth of focus is its integration in three-dimensional optical alignment systems. Other polygonal Fresnel zone plates $[14,15]$ with fractal structure are currently under study.

\section{Acknowledgments}

We acknowledge the financial support from Ministerio de Ciencia e Innovación (grants FIS2011-23175 and TRA2009-0215), Generalitat Valenciana (grant PROMETEO2009-077), and Universitat Politècnica de València (grant PAID-05-11), Spain. 


\section{References}

[1] J.A. Davison, M.J. Simpson, "History and development of the apodized diffractive intraocular lens,” J. Cataract \& Refract. Surg. 32 (2006) 849-858.

[2] S. Wang and X. Zhang, "Terahertz tomographic imaging with a Fresnel lens," Opt. Photon.News 13 (2002) 59.

[3] Y Wang, W. Yun, and C. Jacobsen, "Achromatic Fresnel optics for wideband extreme-ultraviolet and X-ray imaging," Nature 424 (2003) 50-53.

[4] G. Saavedra, W.D. Furlan, and J.A. Monsoriu, "Fractal zone plates," Opt. Lett. 28 (2003) 971-973.

[5] J.A. Monsoriu, G. Saavedra, and W.D. Furlan, "Fractal zone plates with variable lacunarity,” Opt. Express 12 (2004) 4227-4234.

[6] W.D. Furlan, G. Saavedra, and J.A. Monsoriu, "White-light imaging with fractal zone plates," Opt. Letters 15 (2007) 2109-2111.

[7] L.J. Janicijevic, "Diffraction characteristics of square zone plates," J. Optics 13 (1982) 199-206.

[8] J. Alda, J.M. Rico-García, F.J. Salgado-Remacha, and L.M. Sanchez-Brea, “Diffractive performance of square Fresnel zone plates, ” Opt. Commun 282 (2009) $3402-3407$.

[9] H.E. Hart, J.B. Scrandis, R. Mark, and R.D. Hatcher, "Diffraction characteristics of a linear zone plate,” J. Opt. Soc. Am. A 56 (1966) 1018-1019.

[10]W.B. Herrmannsfeldt, M.J. Lee, J.J. Spranza, and K.R. Triggers, "Precision alignment using a system of large rectangular Fresnel lenses,” Appl. Opt. 7 (1968) 995-1005.

[11]F.J. González, J. Alda, B. LLic, and G.D. Boreman, "Infrared antennas coupled to lithographic Fresnel zone plate lenses,” Appl. Opt. 43 (2004) 1-7. 
[12]B. Zhang and D. Zhao, "Square zone plate with spiral phase for generating zero axial irradiance," Opt. Letters 35 (2010) 1488-1490.

[13]E. Maciá, "Exploiting aperiodic designs in nanophotonic devices,” Rep. Prog. Phys. 75 (2012) 036502.

[14]J. Alda and G. Boreman Glenn, "Optimization of polygonal Fresnel zone plates," Microwave Opt. Technol. Lett. 50 (2008) 536-541.

[15]J. Alda and J. Gonzalez "Polygonal Fresnel zone plates," J. Opt. A Pure \& Appl. Opt. 11 (2009) 085707. 